Gigli, A., Demozzi, S. \& Pina-Castillo, M. (2019). La alianza educativa escuela/familia y los grupos de chat de padres: una mirada a la situación italiana. Revista Electrónica Interuniversitaria de Formación del Profesorado, 22(3), 15-30.

\title{
La alianza educativa escuela/familia y los grupos de chat de padres: una mirada a la situación italiana
}

\author{
Alessandra Gigli, Silvia Demozzi, María Pina Castillo \\ Departamento de Ciencias de la Educación. Universidad de Bolonia
}

\section{Resumen}

La llamada a la colaboración entre escuela y familia es una constante de muchos centros educativos, sin embargo, raramente se define su significado y no siempre las medidas de apoyo pragmático son adecuadas, registrándose desde ambas partes situaciones problemáticas de des-alianza. En esta contribución se analizan las coordenadas de la alianza educativa destacando los últimos avances y prefigurando escenarios de resolución desde un punto de vista teórico y metodológico. En particular, se aborda una temática que se presenta cada vez con más fuerza en las dinámicas entre familias y centros educativos: se trata del uso por parte de los progenitores de las nuevas tecnologías de comunicación. Estas nuevas formas de relación, aunque tienen aspectos positivos, se caracterizan por una comunicación poco filtrada, muy inmediata y, a menudo, emocional, repercutiendo a la relación educativa, generando más problemas y haciendo urgente algún tipo de proyecto-acción por parte de las instituciones educativas.

\section{Palabras clave}

Familias; docentes; alianza educativa; nuevas tecnologías de la comunicación; educación.

\section{Educational Alliance school/family and parents' chat groups: a look at the Italian situation}

\begin{abstract}
The call for collaboration between the school and the family is a constant of many educational centers, however, its meaning is rarely defined and not always the measures adopted to support it at the pragmatic level are adequate, registering from both sides
\end{abstract}


problematic situations of des-alliance. In this contribution, the coordinates of the educational alliance are analyzed, highlighting the latest advances and prefiguring resolution scenarios from a theoretical and methodological point of view. In particular, the most adressed topic that appears with more and more force in the dynamics between families and educational and school services: The use of the progenitors of new communication technologies. These new forms of relationship, although they have positive aspects, are characterized by a communication little filtered, very immediate and, often, emotional, having an impact on the educational relationship, generating more problems and urging the need for a type of project-action from the educational institutions.

\section{Key words}

Families; teachers; educational alliance; new communication technologies; education.

\section{Introducción}

En los servicios educativos y escolares italianos se ha extendido la convicción de que la sinergia de intenciones y prácticas entre escuela y familia (alianza educativa) constituya un elemento fundamental para facilitar, amplificar y apoyar el crecimiento de los niños y niñas.

Todavía son pocas las referencias normativas y los documentos oficiales que definen claramente a nivel nacional que significa poner en práctica la alianza educativa; así como son pocas las medidas adoptadas, las inversiones realizadas y las herramientas para apoyarla en la práctica.

Este tema ha sido estudiado en profundidad desde numerosas investigaciones pedagógicas, que desde hace algunos años se han dedicado a monitorear los últimos avances de esta compleja relación (docentes-familias), detectando problemáticas y prefigurando escenarios de resolución, tanto desde un punto de vista teórico como metodológico (Bartolomeo,2004; Contini, 2012; Daffi, 2006; Gigli, 2012, 2016; Lamarca, 2005; y Levorato, 2001).

\section{Situación actual de la alianza educativa: factores de riesgo}

Una primera consideración necesaria es la ambivalencia del sistema escolar, pues desde varios sectores se pide a los docentes ser artífices de un proceso complejo como es el cooperativo, sin embargo, rara vez disponen de suficientes herramientas para llevarlo a cabo.

En general, este compromiso es asumido voluntariamente por el personal educativo, pero no sin dificultad o momentos de crisis. Ambos sujetos protagonistas de la alianza (familias y escuelas) atraviesan un momento caracterizado por fragilidad y se encuentran en la búsqueda de nuevos equilibrios especialmente porque las familias italianas han atravesado auténticas revoluciones antropológicas que han dado lugar al establecimiento de la pluralidad familiar y a la modificación profunda de los estilos educativos en los progenitores. Los fenómenos que caracterizan las dinámicas familiares contemporáneas se pueden resumir en:

- nuevas interpretaciones de los roles materno y paterno con funciones parentales independientes del género del progenitor (Fruggeri, 2011);

- dificultad de las familias para gestionar las funciones normativas y de contención de sus hijos, una vez que el modelo autoritario ha caducado la transición al modelo educativo democrático no se ha completado del todo;

- la crisis que atraviesan las parejas y que pueden derivar en situaciones conflictivas; 
- la vida ajetreada y los trabajos precarios que, en ausencia de políticas adecuadas de bienestar, convierten en oneroso, poder conciliar lo laboral con el cuidado familiar; el uso excesivo de las nuevas Tecnologías de la Información y la Comunicación (TIC) en el entorno familiar y en las relaciones escuela-familia (Gigli, 2016).

Por otra parte, las transformaciones del sistema educativo pueden ser entendidas a partir de los siguientes pasajes clave: clases más numerosas, aumento de la ratio docente/discente; precariedad, menor poder contractual y escasa continuidad laboral de docentes y educadores que disminuye la implicación en proyectos educativos a largo plazo; disminución de la dimensión colectiva del trabajo; aumento de las tareas burocráticas; tendencia general a desvalorizar cultural y económicamente el trabajo docente; afirmación del modelo familiascliente y de la escuela-empresa; cancelación, por insostenibilidad económica, de servicios de apoyo externo (atención pedagógica, terapéutica, etc.); y debilitamiento, a causa de la escasez de recursos, del trabajo en red entre escuela y otras entidades y/o servicios sociales, e impacto con las nuevas emergencias educativas producidas por la dimensión social ampliada (como los fenómenos migratorios).

A pesar de que el escenario que se presenta es muy complejo y poco homogéneo y, a veces, surgen dificultades (conflictos entre docentes y familias, malentendidos, etc.,), casi en toda Italia hay espacios de encuentro y diálogo que activan recursos para mejorar la comunicación escuela-familia; existen oficinas de consulta y garantía de los derechos de familias y alumnado, y el personal está preparado para acoger las peticiones de los progenitores.

Numerosas investigaciones y estudios demuestran que la adecuada participación de las familias en la vida escolar de sus hijos/as puede favorecer el éxito académico, aumentar el bienestar, incrementar la autoestima, mejorar el comportamiento en clase y facilitar las competencias relacionales y sociales (Ballen \& Moles, 1994; Epstein, 1991; García-Carmona, 2015; García, Hernández, Parra y Gomáriz, 2016; Garreta, 2015; Llevot y Bernard, 2015; Poncelet y Kerger, 2010; Silveira, 2016; y Vázquez, López y Colmenares, 2014).

¿Pero qué pasa cuando la participación de la familia no es adecuada? Si la reacción de los docentes es de decepción por la falta de participación, si se desencadenan pensamientos enjuiciantes, ¿qué riesgo corren los niños y niñas? Si la escuela espera de todas las familias las mismas respuestas, se corre el riesgo de que la familia se convierta en un obstáculo adicional para el éxito académico y personal.

Aunque la participación de las familias tiene que ser transversal durante todo el proceso educativo, resulta que el compromiso es menor por parte de los que más lo necesitan, por lo que los centros educativos deben promover una relación de confianza entre docentes y familias (Andrés y Giró, 2016a, 2016b).

Otro riesgo está motivado por el no respeto de los límites del rol, que pueden provocar fenómenos de interferencia o intrusión en las funciones de los docentes, generando dinámicas ambiguas $\mathrm{y}$, a menudo, conflictos.

Finalmente, se detecta un riesgo adicional cuando la presencia de los progenitores se vuelve excesiva y familiariza demasiado el ambiente escolar: volviéndose invasivos, protectores y privando a sus hijos/as de un espacio para conocer e interaccionar de modo autónomo e independiente. Pero ¿cuál es la justa medida?, ¿cuáles son las expectativas adecuadas de la figura del progenitor? y por último, ¿cómo interfieren las TIC en este proceso?

La alianza educativa: representaciones recíprocas, expectativas y fenómenos de des-alianza. 
Contini (2012) pone de relieve la esencia del concepto de alianza, que supone un compromiso en el que dos o más partes asumen una responsabilidad de colaboración con la finalidad de alcanzar una meta conjunta. Lograr este objetivo implica un acuerdo mutuo, elaborar un pacto con un objetivo común: el bienestar y el progreso educativo en niños y niñas/alumnado. Se trata de una relación cooperativa que requiere conocimiento de los roles y de sus límites, confianza mutua en la capacidad del otro para cumplir con sus funciones específicas, solidez de los principios en los que se basa y tolerancia y respeto por la diversidad.

La alianza, por lo tanto, es un proceso complejo. A veces las condiciones necesarias para la alianza no son propicias por varios motivos: carencias del sistema (organizativo o relacional), ausencia de competencias, expectativas erróneas y/o ignorancia, actitud defensiva/agresiva por parte de uno o ambos progenitores (Contini, 2012).

No obstante, estos fenómenos son típicos de la fase inicial de la relación y son probablemente el resultado de una falta de conciencia, ignorancia, una visión corta del proceso educativo, resistencia al cambio, exceso de trabajo y alienación del rol. Obstáculos, éstos, que con mucha dedicación y compromiso pueden ser suavizados o traducidos en relaciones de apoyo mutuo.

La situación mejora, una vez que se consolida la relación tras obtener la confianza del profesorado, y los docentes a menudo se convierten en un recurso para resolver problemas educativos.

Muchas familias recurren a ellos para encontrar soluciones a problemas o sugerencias y consejos, buscando el diálogo, el contacto y el apoyo incluso para problemas extraescolares. Parece que familias y docentes tienen una relación ambivalente, oscilando entre dos polos extremos de desconfianza/confianza. La confianza mutua, piedra angular de la alianza, es un objetivo que la escuela puede conquistar trabajando de manera específica, siguiendo un proyecto pedagógico orientado, preparando herramientas operativas y comunicándose de manera efectiva y pertinente.

Una fortaleza de este discurso es la que establece que la alianza educativa no puede ser un objetivo individual de los docentes, sino una misión de la organización educativa y escolar en su conjunto. Las mejores prácticas de alianza se producen desde una voluntad compartida promovida por un órgano colectivo y dirigente que facilita medios y herramientas y que dirige, apoya y verifica el trabajo del profesorado (Gigli, 2012).

No es objeto de esta contribución profundizar en las coordenadas pedagógicas de la alianza, por lo que nos limitaremos a recordar que es necesario considerar muchos factores que actúan a tres niveles, interdependientes entre sí: dimensión pedagógica, dimensión organizativa, dimensión individual y de trabajo en grupo (Gigli, 2016). En los últimos años, está emergiendo una dimensión adicional en la definición de la relación escuela-familia: el uso de las TIC.

\section{Las nuevas tecnologías de la comunicación y la relación con las familias: el uso de la mensajería instantánea y de los chats.}

Recientemente en Italia, las TIC han entrado en la cotidianeidad de las familias y, por ende, en la relación escuela-familia. Gracias al registro electrónico, los centros educativos pueden poner a disposición de los padres y madres la posibilidad de controlar la asistencia, facilitar las justificaciones on-line, visualizar documentos y comunicaciones y acceder a las evaluaciones. Además, proliferan muy rápido las páginas, los blogs y los foros de información y ayuda mutua dirigidos a los padres y madres (Lupton, Pedersen \& Thomas, 2016) y recurrir 
a las TIC se ha convertido en una tendencia muy común entre los progenitores de la sociedad contemporánea, cuyo éxito quizás se explique por la facilidad de acceso al centro educativo, desde la distancia (Maciá, 2016).

El uso de las TIC en las familias conlleva afrontar, por una parte, aspectos positivos y facilitadores para los niños y niñas, y por otra, algunos negativos relacionados con riesgos y vulnerabilidad (López-Sánchez y García del Castillo, 2017). A pesar de todo, en la actualidad existen pocos estudios sobre el uso de las redes sociales como apoyo en la ardua tarea de la crianza de los hijos e hijas y, aún menos, sobre el uso de las TIC y, en concreto, sobre el uso de la mensajería instantánea entre familia y escuela.

Teniendo en cuenta la presencia de las TIC y lo extendido de su uso, es necesario subrayar algunos datos estadísticos relevantes y algunos puntos clave que se encuentran en la literatura. La investigación ISTAT (2014) sobre Ciudadanos y nuevas tecnologías describe la situación italiana, mostrando un aumento con respecto año anterior del porcentaje de familias que tienen acceso a internet desde casa y aquellos que tienen una conexión de banda ancha (respectivamente desde el $60,7 \%$ al $64 \%$ y del $59,7 \%$ al $62,7 \%$ ).

Internet se caracteriza como un importante instrumento para la interacción social, siendo la principal consecuencia el alto grado de expansión de los smartphones (Pereira-Da Silva y Burgos-Rocha, 2017), pues ocho de cada 10 internautas lo han utilizado en los últimos tres meses para recibir o enviar un correo, el 65,7\% para enviar mensajes en chat, blog, foros de discusión online o por mensajería instantánea, más de la mitad (57\%) para enviar mensajes a través de Facebook o Twitter. En el ámbito de la utilización de las diversas plataformas comunicativas disponibles en la red, respecto al 2013 se registra un leve ascenso en el uso del correo a favor de otras formas de interacción, como el uso de las redes sociales (del 53,2\% de 2013 al 57\% de 2014) y el uso de la mensajería en chat, blog, grupos de noticias o foros de discusión online (del 49\% del 2013 al 50\% del 2014).

Para el uso de la mensajería con WhatsApp (WA) debemos hacer una referencia a datos a escala internacional, hipotetizando un alineamiento de Italia con otros países occidentales. WA representa uno de los instrumentos principales de entretenimiento entre individuos (O'Hara, Massimi, Harper, Rubens \& Morris, 2014) y comparando el uso de WA y SMS, el primero se usa muchísimo más tanto para comunicarse dentro de círculos sociales restringidos como para comunicaciones de grupo como los chats (Church \& de Oliveira, 2013). Por otra parte, Sultan (2014) afirma que los usuarios de WA prefieren esta aplicación para mantenerse en contacto con amigos y familiares, pero también, en general, para obtener información. El uso de la mensajería instantánea, parece ser un medio privilegiado en las interacciones no formales para mantenerse actualizado y en contacto con amigos y familiares, tanto es así, que casi ha suplantado las interacciones cara a cara (Harrison \& Gilmore, 2012).

A nivel internacional, las pocas investigaciones sobre familias y el uso de las TIC, muestran una preferencia por parte de los padres y madres en el uso de una comunicación basada en Internet cuando buscan apoyo y comparación entre iguales (Metzler, Sanders, Rusby \& Crowley, 2012 y Sanders, Haslam, Calam, Southwell \& Stallman, 2011). El uso de las TIC en contextos educativos es cada vez más evidente, también en la comunicación entre las familias y los centros educativos e incluso entre las propias familias. En particular, emergen, junto a los canales tecnológicos oficiales (previstos por la propia escuela) también grupos en varios ambientes online a través de aplicaciones para la comunicación instantánea (WA, Messenger, Facebook, Telegram o similares). Además, están los blogs de los padres y madres o las páginas de Facebook creadas, siempre por las familias, para hacer pública la actividad del centro educativo. 
Estos grupos para-oficiales se crean con el objetivo de facilitar el intercambio de comunicación entre los representantes de clase y otros padres, sin embargo, muy a menudo, se transforman en entornos de intercambio de otras temáticas: como las impresiones sobre el profesorado, las narraciones de episodios contados por los niños y niñas, solicitudes de información sobre tareas, enfermedades, pasando por problemas organizativos de diversa índole, hasta llegar a cuestiones personales, dificultades, aspectos relacionados con las relaciones entre niños y niñas o entre familiares. No solo comunicaciones funcionales, por lo que la intromisión es muy frecuente y no está exenta de peligros y dificultades.

Los docentes rara vez participan en estos grupos, donde se habla mucho sobre ellos, pero sin poder controlar la calidad de las opiniones expresadas. No es difícil imaginar cómo estas nuevas formas de comunicación afectan a la alianza educativa: creando e intensificando el intercambio entre padres en un entorno de comunicación muy particular caracterizado por una comunicación poco filtrada, muy inmediata y a menudo emocional, donde se crea un mundo paralelo en el que el sujeto escuela no tiene acceso. En realidad, este mundo paralelo siempre ha existido, en los patios, en los jardines, en los grupos de padres y madres fuera de las escuelas, aunque hoy en día es un fenómeno diferente, en el que la comunicación viaja a una velocidad mucho mayor y en una modalidad menos exigente que la comunicación directa.

A veces esta participación comunicativa se manifiesta de una manera particularmente intensa, los mensajes y las respuestas son muchos y todos los días, se mandan fotos, opiniones, se alimentan inquietudes y preocupaciones, los problemas se magnifican, cuestiones diversas qué en realidad, merecerían menos atención y energía, existiendo un interés por aspectos que incumben a los niños y niñas y, a menudo, se juzgan las acciones de los docentes evitando hablar directamente con ellos.

Muchos padres y madres, por supuesto, preferirían quedarse al margen, no obstante, el miedo de aislarse y aislar a sus hijos/as los obliga a una participación forzada. Este caso la literatura lo define como FOMO (Fear of Missing Out) miedo a quedarse fuera, una especie de temor a permanecer separado de lo que los otros comunican en estos entornos virtuales (Przybylski, Muryama, DeHaan \& Gladwel, 2013).

Como resultado, no solo los sujetos permanecen en contextos comunicativos en los que no participarían voluntariamente, sino que mantienen una conexión continua y constante, de forma casi obsesiva. Otros encuentran espacios de pertenencia y relación que de otra manera no tendrían, Ilenando un vacío relacional personal a través de una forma social mediada por la educación de sus hijos/as.

\section{Metodología}

A la luz de estas últimas consideraciones, en 2017 se diseña el proyecto de investigación exploratoria "Padres y TIC: como las nuevas tecnologías de comunicación influyen en los estilos educativos parentales y la alianza educativa", que tiene como objetivo recoger información sobre el uso que las familias italianas hacen de las TIC en general, y más en particular, la relación con sus obligaciones como padres y madres, individualmente y en el intercambio con otros progenitores y con los docentes. El proceso de aplicación se realizó a través de una encuesta en línea en google drive.

La investigación completa (con el análisis de todos los datos recogidos) está en proceso de publicación (Cino, Gigli y Demozzi, 2019 y Gigli, 2019); aquí se presentan solo algunos resultados relativos al intercambio comunicativo escuela-familia mediante las TIC. 
Se diseña y se aplica un cuestionario semiestructurado online compuesto por 30 ítems de diferentes tipos, de los cuales 10 corresponden a variables socioeconómicas de respuesta cerrada y 20 al uso de las TIC ( 16 de respuesta cerrada, dos semiabierta y dos abierta dividas en; hábitos de conexión; participación en grupos de Facebook; participación en grupos de chat; sharenting; y uso de las TIC entre profesorado y padres). Los datos cuantitativos fueron analizados con SPPS Para todas las pruebas estadísticas (correlación, regresión y Chi cuadrado) se estableció un nivel de significación estándar (nivel alfa) de .05.

Sin embargo, aquí presentamos solamente algunos datos puramente descriptivos relacionados con el uso de los chats de WA y de las tecnologías en la relación profesoradopadres y no se presentan (excepto en un caso) ni correlaciones ni regresiones.

El cuestionario se aplicó a una muestra no probabilística formada por progenitores italianos que accedían a cumplimentar el cuestionario que se compartía en tres páginas de Facebook de grupos de padres, una vez había sido aceptada su solicitud formal por parte de los administradores.

El estudio se realizó garantizando el anonimato y el cumplimiento de la ley italiana actual sobre privacidad y protección de datos confidenciales.

La encuesta fue llevada a cabo con éxito por una muestra de 302 encuestados, la mayoría de los cuales representada por madres (85\%). Los grupos de edad más representativos son entre 41-50 años (49\%) y 31-40 años (37\%). La muestra está representada por padres con un nivel educativo alto: el $71 \%$ tiene un título universitario y el $89 \%$ tiene empleo. El número medio de hijos por familia es de dos. Los análisis se llevaron a cabo considerando los datos relativos solo al primer hijo. La edad de los primogénitos estaba distribuida de la siguiente forma: $16 \%$ de menores entre 0-3 años; $20 \%$ entre 4-6; el 18\% entre 7-9; $14 \%$ entre $10-12$ y el 32\% mayor de 13 años.

\section{Resultados}

\section{Uso de los chats de WhatsApp de la muestra analizada}

Los resultados obtenidos reflejan que los padres forman parte de 2.57 grupos, con un rango de uno a 11 grupos, en cuanto a la frecuencia el $85,44 \%$ de las mujeres y el $61,11 \%$ de los hombres utiliza WA u otros sistemas de mensajería más de una vez al día, como se puede ver en el siguiente gráfico de forma más detallada:

Por otro lado, de promedio el $80 \%$ usa WA, de los cuales el $45 \%$ participa activamente en los chats, el $30 \%$ simplemente lee los mensajes, el $25 \%$ declara participar a regañadientes, pero prefiere no quedarse fuera, como se aprecia a continuación:

Existe una correlación significativa entre el número de niños y el número de grupos de WA, por lo que los progenitores con más hijos están inscritos en más de un grupo (los datos resultantes de la correlación de Pearson $r(240)=.34, p<.001$ ), de los cuales: $53 \%$ son miembros de dos-tres grupos, $26 \%$ de más de tres grupos, y $21 \%$ de un grupo.

De los resultados se desprende, además, que las madres tienen una probabilidad cuatro veces mayor que los padres de ser miembros de al menos un grupo de WA; y que los progenitores de entre 41 a 50 años tienen una probabilidad tres veces superior de formar parte de un grupo de WA con respecto a los padres y madres más mayores. Con relación al número de hijos/as, los progenitores con más de uno tienen una probabilidad tres veces superior que aquellos de hijas/as únicos/as a formar parte de estos grupos. 


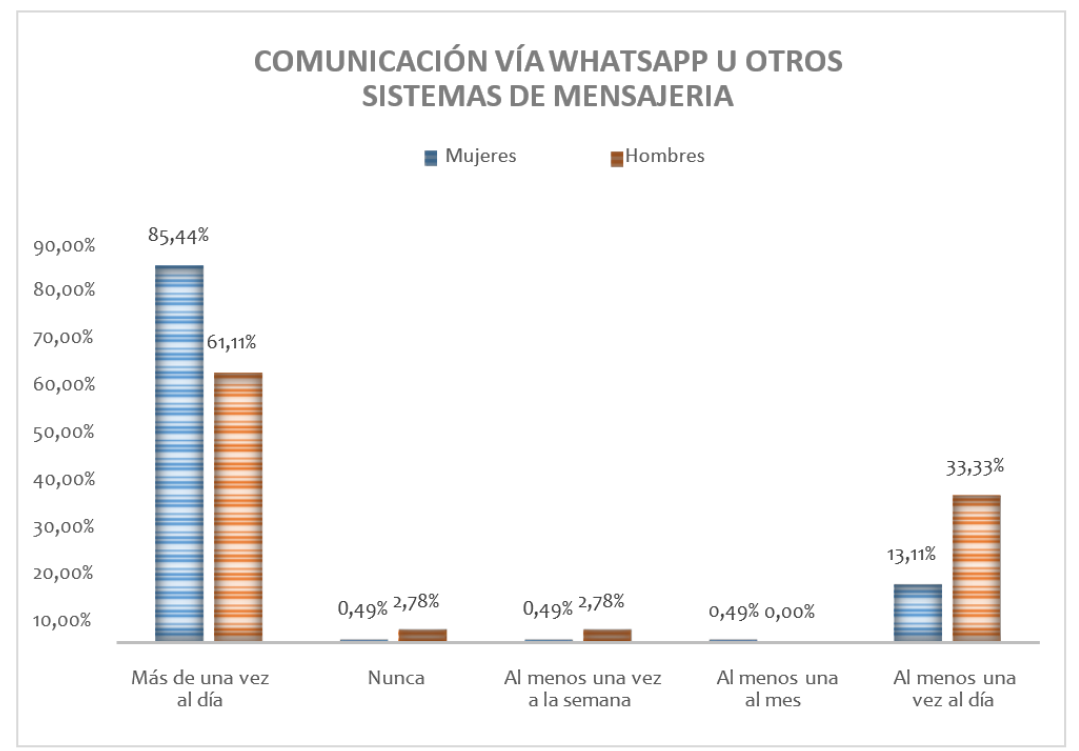

Figura 1. Uso de WA u otros sistemas de mensajería

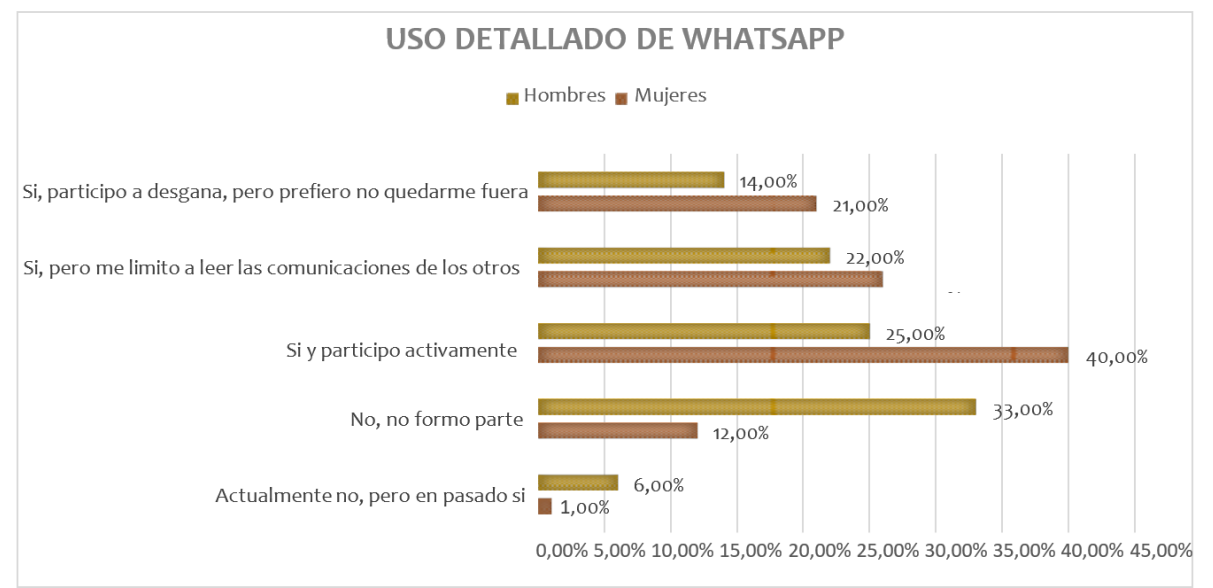

Figura 2. Uso detallado de WA

En una de las preguntas abiertas del cuestionario, los progenitores participantes debían expresar su opinión en relación con el uso de los chats entre grupos de padres. De los resultados recogidos de los participantes la opinión más extendida es que son muy útiles para el intercambio de información útil (84\%). Este intercambio, sin embargo, parece ser meramente informativo, ya que el $65 \%$ de la muestra cree que dentro de los grupos de chat de progenitores no hay lugar para compartir problemas y encontrar consuelo, el 79\% también cree que los grupos de chat no son espacios adecuados para socializar ni conocer las opiniones de los otros.

En cuanto a la probabilidad de obtener información sobre sus hijos, la muestra se divide sustancialmente, el $55 \%$ no esta de acuerdo y $45 \%$ si. El 50\% de los progenitores cree que estos chats suponen una pérdida de tiempo y el $60 \%$ esta de acuerdo en que las personas que participan lo hacen a desgana.

En la siguiente figura, se puede apreciar la opinión de los participantes que declaran hacer parte de los chats, en concreto 202 progenitores: 


\section{¿HAS ABANDONADO ALGUNA VEZ ESTOS}

\section{CHATS?}

no, pero me hubiera gustado a no, nunca he sentido esa necesidad $\quad$ Si

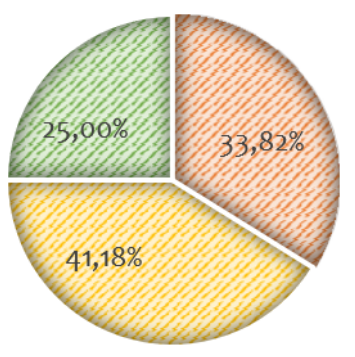

Figura 3. Abandono de los grupos de chats

\section{Las TIC y la comunicación entre familias-docentes}

Entre las herramientas más utilizadas en la comunicación escuela-familia en la muestra objeto de estudio, predominan el registro electrónico (47\%), la página web de la escuela (46\%) y el intercambio a través de listas de correo (35\%). El uso de la mensajería instantánea y las redes sociales es una minoría, el chat entre progenitores y docentes está presente en un 13\%, mientras que la presencia de una página de Facebook es del 10\%. En el siguiente gráfico se puede valorar el reparto, teniendo en cuenta que se trata de una pregunta con varias alternativas de respuesta:

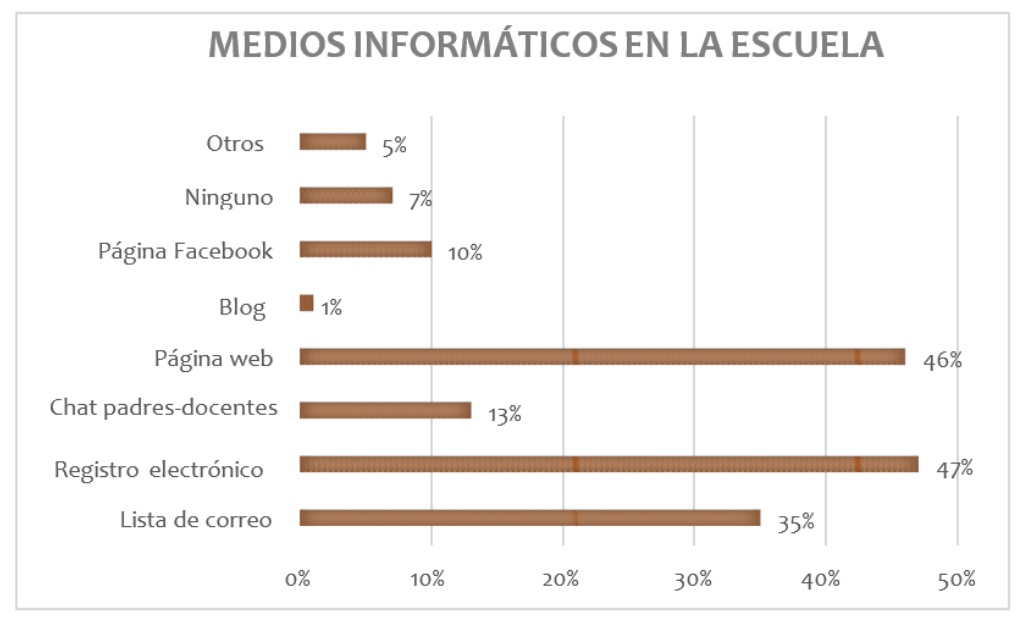

Figura 4. Medios informáticos en la escuela

Entre los cursos escolares analizados, la manifestación de intercambios importantes a través del chat tiene lugar en la escuela primaria (32\%), seguida de infantil y guarderías (ambas en el 
19\%). A continuación, una síntesis de los instrumentos más utilizados para la comunicación escuela-familia, según el nivel educativo:

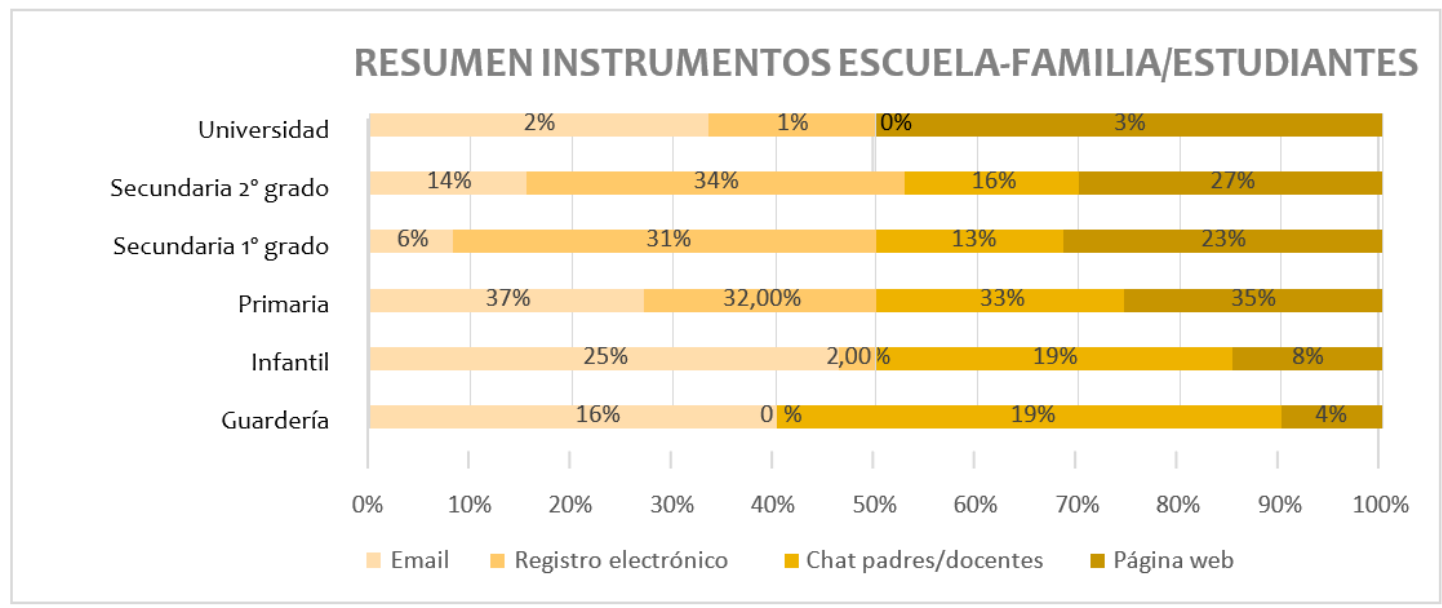

Figura 5. Instrumentos según nivel educativo

El escaso uso de la mensajería instantánea con los docentes ha demostrado también el hecho de que, según el $94.7 \%$ de los progenitores participantes, el profesorado está bastante/muy dispuesto a comunicarse en entrevistas presenciales. La disposición a comunicarse a través de medios diferentes, por otro lado, es diametralmente opuesta: los docentes están muy dispuestos a comunicarse por teléfono con el número personal en el $29 \%$ de los casos. Más disponibles en la escuela primaria (38\%), seguido por la guardería (37.3\%) y los centros de secundaria (24\%). También se reduce la disponibilidad para comunicarse a través de SMS con el número personal (el $74.5 \%$ de los docentes tienen poca o ninguna disponibilidad); se afirma una mayor disponibilidad en la escuela primaria (35,9\%). En cuanto a que los docentes compartan el número de teléfono personal, la mayoría de las familias no están muy de acuerdo, probablemente por el respeto de la privacidad ( $74.9 \%$ es muy/bastante de acuerdo en que no es correcto: con una ligera mayoría en el centro de Italia (80\%) en el norte (75\%) en comparación con el sur (66.7\%). A nivel de la escuela primaria se constata también hay una mayor disposición a comunicarse por correo electrónico (44.6\%, aproximadamente el doble que en otras etapas escolares). La gran mayoría de los docentes no está disponible para comunicarse, ni en salas de chat administradas por las familias ni por docentes (90.4\%). Se registra una predisposición muy baja también en el caso de los grupos de Facebook (95.7\% para nada disponible). En los pocos casos en los que existía predisposición a comunicarse ya sea por chat como por Facebook, la disponibilidad se registraba en el sur del país.

Cuando se preguntó a las familias si, más allá de su uso real, todavía consideran importante que los docentes estén disponibles para comunicarse a través del chat u otros tipos de mensajería instantánea, la muestra se divide de la siguiente manera: en el rango de progenitores con hijos menores de tres años, el 19.9\% cree que es bastante/muy importante; en el grupo de tres a seis años, el $22,3 \%$ cree que sí y en el grupo de educación primaria el $25,3 \%$, mientras que en secundaria el $26,2 \%$. Los datos, por lo tanto, crecen con el aumento del grado escolar también porque, probablemente, disminuyen las ocasiones de interacción diaria con los docentes. 


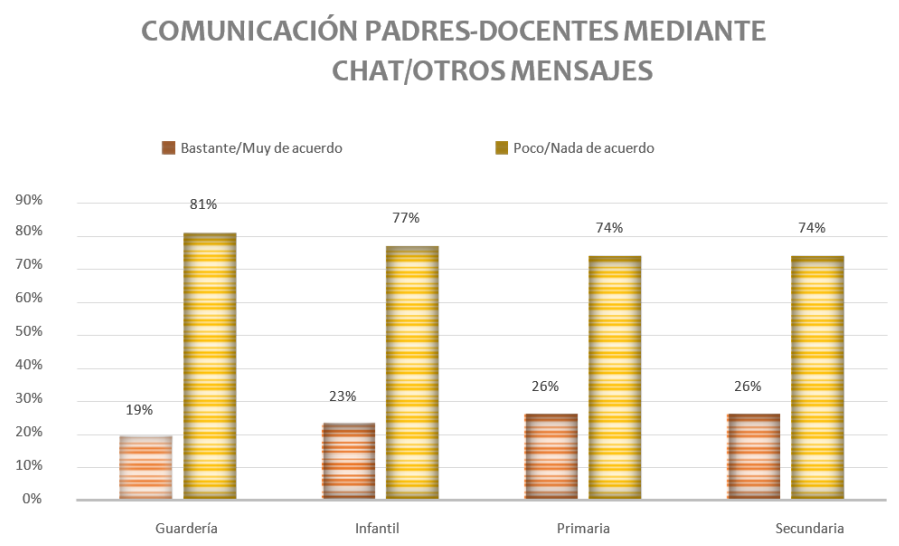

Figura 6. Comunicación padres-docentes mediante chat

En general a toda la muestra le gustaría tener más reuniones presenciales con los docentes de sus hijos/as (80\%). En caso de que los niños experimenten malestar relacionado con a la vida escolar, el $41 \%$ de la muestra intenta hablar en persona con el profesor o profesora y el $32 \%$ escribe al docente para concertar una reunión. Un 16\% recurre a otros progenitores o miembros de la familia (a menudo por teléfono o mensaje) y solo el $3 \%$ utiliza el grupo de chat. Entre los que intentan hablar en persona con el docente, el 35\% son familiares de niños de secundaria, mientras que el $25 \%$ son de segundo grado y el $22 \%$ de primaria. En el caso de solicitud por escrito para una entrevista, el $36 \%$ son progenitores de escuela primaria, el $29 \%$ de la escuela secundaria de primer grado y el $25 \%$ de segundo grado. El porcentaje de los progenitores que recurren a chats o grupos es bajísimo y la mayoría es de secundaria de segundo grado (21\%).

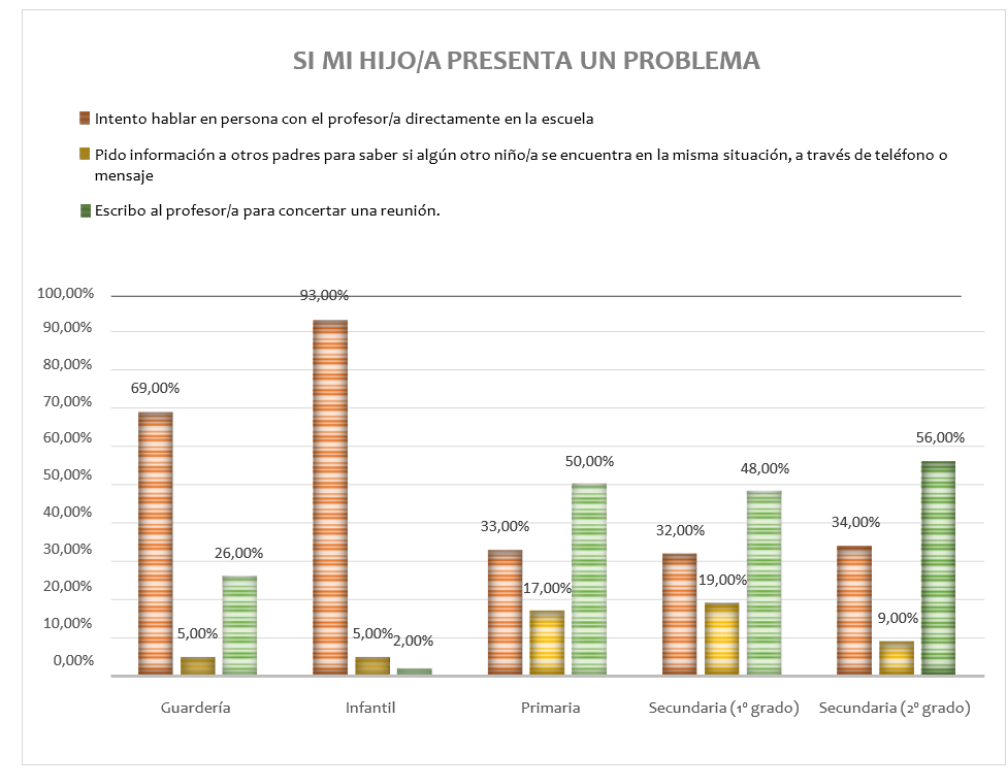

Figura 7. Si mi hijo/a presenta un problema.

En el caso de problemas con la entrega de las tareas, el 41\% de los progenitores participantes de la muestra hacen uso de los grupos de chat y el $35 \%$ siempre les pide información a otros 
progenitores por teléfono o mensaje. Se reduce drásticamente el recurso a entrevistas directas con el profesorado (16\%) y el uso del correo electrónico con el docente (7\%).

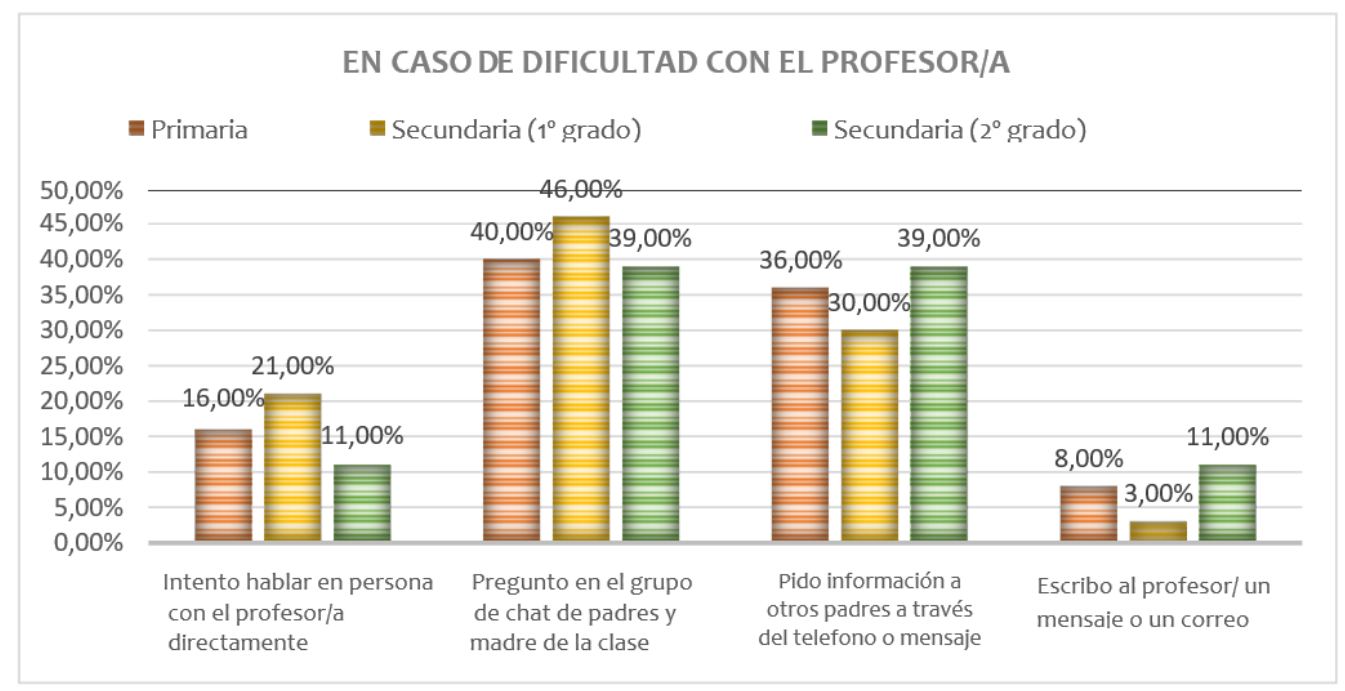

Figura 8. En caso de dificultad con el profesor/a

Parece, por lo tanto, que las familias participantes recurren al uso de mensajes instantáneos solo para el intercambio de información, especialmente con respecto a tareas y/o actividades educativas. En cuanto a problemas más serios y exigentes, se manifiesta a todos los niveles la necesidad de que las familias tengan una entrevista cara a cara con los docentes. Esta necesidad aumenta con el aumento del curso escolar probablemente también porque, las ocasiones de encuentro directo disminuyen. Esto además, cuestiona la dificultad de las familias para confiar en sus hijos/a y apoyar su nivel de autonomía en el manejo de la vida escolar, de hecho, al crecer, los niños deben representar el canal directo de comunicación entre escuela-familia y entre progenitores. A medida que el niño progresa en su carrera escolar, los progenitores deben tratar de disminuir su protagonismo e interferencia directa en la alianza educativa.

\section{Discusión y conclusiones}

De los datos que emergen de la investigación, surgen los siguientes elementos de riesgo que pueden dificultar la relación escuela/familia como la intromisión y el uso excesivo y compulsivo de estas herramientas por parte de algunos individuos, que a su vez pueden crear círculos viciosos de comunicación que corren el riesgo de generar prejuicios, estereotipos, invasiones e incluso la violación de algunas reglas de privacidad. Existen algunos casos de acciones legales en los cuales los intercambios comunicativos que tuvieron lugar en estos grupos o en las redes sociales se utilizan como prueba de culpabilidad precisamente por difamación y/o violación de la privacidad.

Los profesionales educativos permanecen al margen, pero constatan en sus aulas cómo el uso incorrecto de estos instrumentos tiene consecuencias, incluso muy graves, sobre las dinámicas entre alumnado y profesorado. Con este escenario y esta problemática es muy importante que la escuela o el servicio estén dotados de herramientas apropiadas 
(organizativas, normativas y de competencias técnicas) para administrar directamente los blogs o las páginas oficiales en las redes sociales, con el fin de intentar evitar malentendidos, incomprensiones o incluso conflictos derivados del uso incorrecto de estas tecnologías.

La única forma de intervención actualmente posible parece ser la prevención que se debe implementar con urgencia en las primeras fases de bienvenida a las familias. En primer lugar, se trata de informar a los padres sobre los riesgos reales de un uso incorrecto de estas herramientas de comunicación, de los posibles efectos en términos educativos y relacionales, así como legales. En consonancia los progenitores solicitan más reuniones presenciales con los docentes de sus hijos/as, especialmente en caso de problemas escolares, al mismo tiempo, los docentes muestran una apertura y disponibilidad para comunicarse con las familias. Muchas escuelas y servicios educativos, ante esta fuerte demanda de reuniones cara a cara, intentan organizar más momentos de encuentro en horarios que sean compatibles con el trabajo de los progenitores y garantizando también un espacio supervisado para sus hijos durante la reunión. Especialmente en los servicios educativos dedicados a la etapa de edad 0-6 años, la cultura del cuidado de la relación con los padres está muy presente (supervisada por un cuidadoso trabajo de pedagogos), que prioriza el contacto directo y trata de desincentivar en la medida de lo posible la comunicación mediada por la TIC, y en particular por los chats.

A pesar de la tendencia al uso siempre más presente de las TIC en la vida cotidiana, a día de hoy en Italia las instituciones educativas públicas no han activado medidas particulares de intervención porque aparentemente, y esto también es lo que emerge de nuestra primera investigación exploratoria, esto todavía afecta muy poco a los intercambios de comunicación entre la escuela y la familia, prevaleciendo un intercambio simple de información (sobre todo a través del sitio web de la escuela/servicio y el registro electrónico).

Sin embargo, se considera importante, siempre que sea posible, que las escuelas enfaticen la importancia de la comunicación no mediada, desalentando el uso del chat, así como la necesidad de asumir un comportamiento cuidadoso y consciente del uso de las tecnologías en contextos comunitarios. Resulta recomendable invitar a los padres a comunicarse lo más directamente posible con los docentes, a respetar la privacidad, a abstenerse de comentarios inapropiados sobre otros adultos y especialmente sobre los menores, a verificar la información antes de comentarla, a filtrar los mensajes que ignoran los estímulos que no parecen fundamentales e inherentes a su papel en la vida escolar de los niños y niñas. El uso de las nuevas tecnologías en contextos educativos requiere el establecimiento de una normativa que regule la interacción familia-escuela (Fernández, Yañez y Muñoz, 2015; Garreta, 2015; Sánchez y Cortada, 2015). Algunos servicios y escuelas sí que tienen un decálogo para el uso correcto de las TIC: una especie de netiqueta para uso escolar (un término que combina la palabra inglesa net, red y la palabra francesa buena educación, etiquette), una especie de protocolo que, de ser respetado y aplicado con sentido común, puede sacar el lado positivo y útil de estos medios.

Además, dada la gran importancia de las reuniones en persona, resulta oportuno continuar invirtiendo sobre la formación (inicial y continua) de los docentes y del personal educativo con respecto a sus habilidades comunicativas y relacionales, en la dirección d.e una comunicación siempre más eficaz, empática, capaz de abrir la puerta a las relaciones de confianza mutua, estima y de compartir objetivos educativos. Sin perder de vista el tema de las TIC, cuya presencia comienza a ser siempre más real incluso en la relación escuela-familia.

Para concluir, es importante poner de relieve que el mundo de la educación y los centros educativos ya no pueden permanecer al margen o desinteresarse de estos temas porque, 
quieran o no, son elementos constitutivos de la forma de vivir en comunidad y, por tanto, piezas fundamentales en cualquier proyecto educativo.

\section{Referencias}

Andrés, S. y Giró, J. (2016a). La participación de las familias en la escuela: una cuestión compleja. Revista de Evaluación de Programas y Políticas Públicas. 7 (1), 28-47. doi: 10.5944/reppp.7.2016.16302

Andrés, S. y Giró, J. (2016b). El papel y la representación del profesorado en la participación de las familias en la escuela. Revista Electrónica Interuniversitaria de Formación del Profesorado, 19 (1), 61-71. doi: 10.6018/reifop.19.1.245461.

Ballen, J. \& Moles, O. (1994). Strong families, strong schools: building community partnerships for learning. Washington, U.S: Department of Education.

Bartolomeo, A. (2004). La relazione genitori insegnanti. Brescia, Itallia: la Scuola.

Contini, M.G. (2012). Dis-alleanze nei contesti educativi. Roma, Italia: Carocci.

Church, K. \& Oliveria, R. (2013) What's up with Whatsapp? Comparing Mobile Instant Messaging Behaviors with Traditional SMS. In 15th International Conference on Human-Computer Interaction. Mobile $\mathrm{HCl}$, Munich, Germany.

Cino, D., Gigli, A. \& Demozzi, S. (2019). That's the only place where you can get this information today! An exploratory study on Parenting WhatsApp Groups with a sample of Italian parents. Artículo entregado para la publicación.

Daffi, G. (2006). Genitori in clase. Bari, Italia: la Meridiana.

Epstein, J. L. (1991). Effects on student achievement of teachers' practices of parent involvement, in S.B. Silbern (Ed), Advances in reading and language research: a research annula, Vol. 5. Literacy through family, community, and school interaction (pp. 261-276). US: Elsevier Science/JAI Press.

Fernández, J.M., Yáñez, J.A. y Muñoz, E.K. (2015). Prácticas morales y normas de netiqueta en las interacciones virtuales de los estudiantes de educación secundaria. Innovación educativa, 15 (69), 57-72.

Fruggeri, L. (2011). Genitorialita dall'atribuzione del ruolo all'esercizio di una funzione. In Gigli, a. (Ed), Maestra, ma Sara ha due mamme? Le famiglie omogenitoriali nei servizi scolastici e educativi, (pp.66-77). Italia, Milano: Guerini Scientifica.

García, M.P., Hernández, M.A., Parra, J., y Gomáriz, M.A. (2016). Participación familiar en la etapa de educación primaria. Perfiles educativos, 38(154), 97-117

García-Carmona, M. (2015). Las TIC como herramientas facilitadoras de la participación de las familias en las asociaciones de madres y padres. En. Jabonero, M., Martín Bris, M., Martínez Arias, A., y Bizelli, J.L. (Ed), Miradas diversas de la educación en Iberoamérica (pp. 399-412). Madrid, España: Universidad de Alcalá.

Garreta , J. (2015). La comunicación familia-escuela en Educación Infantil y Primaria. Revista de la Asociación de Sociología de la Educación, 8 (1), 71-85

Garreta, J. (2016). Fortalezas y debilidades de la participación de las familias en la escuela. Perspectiva educacional, 55(2), 141-157. doi: 10.4151/07189729 
Gigli, A. (2012). L'alleanza ai tempi della crisi: elementi di problematicità e fattori propulsivi. En Contini. M.G. (Ed), Disalleanze nei contesti educativi (pp. 35-48) Roma, Italia: Carocci.

Gigli, A. (2016) Famiglie evolute. Capire e sostenere le funzioni educative delle famiglie. Italia: Edizioni Junior.

Gigli, A. (2019) Information and Communication Technologies in school-family communication and parental involvement in children's schooling: data from a research. Artículo entregado para la publicación.

Harrison, M.A. \& Gilmore, A. L. (2012). U TXT when? College students' social contexts of the text messaging. The Social Science Journal, 49, 513-518. doi: 10.1016/j.soscij.2012.05.003

Istat (2014). Cittadini e nuove teconologie. Italia: Instituto Nazionale di Statistica. Recuperado de: www.istat.it/it/files//2014/12/Cittadini_e_nuove_tecnologie_anno-2014.pdf

La Marca A. (2006). Famiglia e scuola. Roma, Italia: Armando Editore.

Levorato, R. y Levorato, A. (2011). La famiglia incontra la scuola. Roma, Italia: San Paolo.

Llevot, N. y Bernard, O. (2015). La participación de las familias en la escuela: factores clave. Revista de la Asociación de Sociología de la Educación, 8 (1), 57-60.

López-Sánchez, C. y García del Castillo, J.A. (2017). La familia como mediadora ante la brecha digital: repercusión en la autoridad. Revista Colombiana de Ciencias Sociales, 8 (1), 108.124. doi: 10.21501/22161201.1928

Lupton, D., Pedersen, S., \& Thomas, G.M. (2016). Parenting and digital media: from the early web to contemporary digital society. Sociology Compass. 10 (1): 730-743. doi: 10.1111/soc4.12398.

Macià, M. (2016). La comunicación familia-escuela: el uso de las TIC en los centros de primaria. Revista Electrónica Interuniversitaria de Formación del Profesorado, 19(1), 73-83. doi: 10.6018/reifop.19.1.245841.

Metzler, C.W., Sanders, M. R., Rusby, J.C. \& Crowley, R.N. (2011). Using consumer preference information to increase the reach and impact of media-based parenting interventions in a public health approach to parenting support. Behav Ther, 43 (2), 257-270. doi: 10.1016/j.beth.2011.05.004

Pereira-Da Silva, I., y Burgos-Rocha, F. (2017). Implicações do uso do whatsapp na educação. Revisa EDaPECi, 17 (2), 161-174

Przybylski, A.K., Muryama, K., DeHaan, C.R. \& Gladwell, V. (2013). Motivational, emotional, and behabioral correlates of fear of missing out. Computers in Human Behavior, 29 (4), 1841-1848. doi: 10.1016/j.chb.2013.02.014

Poncelet D., y Kerger S. (2010). Le point sur l'éducation familiale et les relations école-famille au Luxembourg, in E. Catarsi (Ed), La formation et la recherche en éducation familiale. Etats des lieux par pays, (pp. 31-48). Pisa, Italia: Edizioni del Cerro.

Sánchez, I. \& Cortada M. (2015). Digital resources in the family-school relationship during the 0-3 stage. Cultura y Educación, 27 (1), 221-233. doi: 10.1080/11356405.2015.1006851

Sanders, M.R., Baker, S. \& Turner, K.M. (2012). A randomized controlled trial evaluating the efficacy of Triple $P$ Online with parents of children with early-onset conduct problems. Behaviuour Research and Therapy, 50 (11), 675-684. doi: 10.1016/j.brat.2012.07.004 
Sanders, M.R., Haslam, D.M., Calam, R., Southwell, C., \& Stallman, H. M. (2011) Designing effective interventions for working parents: A web-based survey of parents in the UK workforce. Journal of Children's Services, 6 (3), 186-200. doi: 10.1108/1746666

Silveira, H. (2016). La participación de las familias en los centros educativos. Un derecho en construcción. Revista Electrónica Interuniversitaria de Formación del Profesorado, 19 (1), 17-29. doi: 10.6018/reifop.19.1.245511

Sultan, A.J. (2014). Addiction to mobile text messaging applications is nothing to "lol" about. The Social Science Journal, 51 (1), 57-69. doi: 10.1016/j.soscij.2013.09.003

O’Hara, K., Massimi, M., Harper, R., Rubens, S \& Morris, J. (February, 2014). Everyday Dwelling with WhatsApp. In ACM Conference on Computer Supported Cooperative Work, Mobile Apps for Enhancing Connectedness, Baltimore, US.

Vázquez, E., López, E. y Colmenares, L. (2014). La dimensión socializadora de la tecnología para una comunidad educativa más abierta y colaborativa. Revista de Tecnología de Información y Comunicación en Educación, 8 (1), 145-157. 